\title{
A Necessary and Sufficient Condition for Hardy's Operator in the Variable Lebesgue Space
}

\author{
Farman Mamedov ${ }^{1}$ and Yusuf Zeren ${ }^{2}$ \\ ${ }^{1}$ Mathematics and Mechanics Institute of NAS and OGRDI of SOCAR Company, 9 B. Vahabzade, 1141 Baku, Azerbaijan \\ ${ }^{2}$ Department of Mathematics, Yildiz Technical University, Esenler, Istanbul, 34220 Davutpasha, Turkey
}

Correspondence should be addressed to Farman Mamedov; farman-m@mail.ru and Yusuf Zeren; yzeren@yildiz.edu.tr

Received 11 September 2013; Revised 28 March 2014; Accepted 9 April 2014; Published 6 May 2014

Academic Editor: Vakhtang M. Kokilashvili

Copyright (C) 2014 F. Mamedov and Y. Zeren. This is an open access article distributed under the Creative Commons Attribution License, which permits unrestricted use, distribution, and reproduction in any medium, provided the original work is properly cited.

The variable exponent Hardy inequality $\left\|x^{\beta(x)-1} \int_{0}^{x} f(t) d t\right\|_{L^{p(.)(0, l)}} \leq C\left\|x^{\beta(x)} f\right\|_{L^{p(.)}(0, l)}, f \geq 0$ is proved assuming that the exponents $p:(0, l) \rightarrow(1, \infty), \beta:(0, l) \rightarrow \mathbb{R}$ not rapidly oscilate near origin and $1 / p^{\prime}(0)-\beta>0$. The main result is a necessary and sufficient condition on $p, \beta$ generalizing known results on this inequality.

\section{Introduction}

There are a lot of examples in the theory of differential equations and analysis, in which the boundedness of the Hardy operator in some spaces is used essentially (a weighted Lebesgue space, Lorentz space, their weak spaces, and so on; see, e.g., [1-4]). The aim of present paper is to study a necessary and sufficient condition for the boundedness of Hardy operator in the weighted Lebesgue space $L^{p(\cdot)}(0, l)$ with variable exponent $p:(0, l) \rightarrow(1, \infty)$. The investigation of variable exponent Lebesgue spaces is stimulated with the modeling of electrorhelogical fluids (see $[5,6]$ ). That led to the development of regularity theory for the nonlinear elliptic and parabolic equations with partial derivatives (see, e.g., the bibliography in $[7,8])$. Also, the required mathematical methods of analysis were elaborated to study the boundedness of principal integral operators (maximal operator, fractional operators, singular operator, commutators, and so on) in spaces $L^{p(\cdot)}$ (see the recent monographs $\left.[9,10]\right)$.

In all probability, the first investigation of the variable exponent Hardy inequality was started in the works [11$15]$, subsequently in [16-20]. The variable exponent Hardy inequality was considered also in the recent works [2125]. Since the Hardy operator is the simplest one among other integral operators, it seems logical to investigate the necessary and sufficient conditions for this operator in the first place. Though we have not so far succeeded in obtaining appropriate results in the general weighted space and in the case of general exponential functions, the representation of our results in presenting here form seems to us more attractive for comparision with the known results (see below).

More precisely, the subject of present paper is to study the norm inequality

$$
\left\|x^{\beta(x)-1} H f\right\|_{L^{p(\cdot)}(0, l)} \leq C\left\|x^{\beta(x)} f\right\|_{L^{p(\cdot)}(0, l)} ; \quad f \geq 0
$$

for the Hardy operator

$$
H f(x)=\int_{0}^{x} f(t) d t
$$

Due to the cited above results, a necessary and sufficient condition for (1) take place if a regularity condition is assumed on $p, \beta$ at the origin. Namely, let $1<p^{-}=\inf p$, $p^{+}=\sup p<\infty,-\infty<\beta^{-}, \beta^{+}<\infty$, both functions $p, \beta$ satisfy the condition

$$
\limsup _{x \rightarrow 0}|g(x)-g(0)| \log \frac{1}{x}<\infty
$$

then inequality (1) holds if and only if $\beta(0)<1 / p^{\prime}(0)$. 
In our results, the exponent functions $p(\cdot), \beta(\cdot)$ satisfy the following oscillation condition near origin:

$$
\limsup _{x \rightarrow+0}\left[\sup _{|y-x|<x / 2}|g(x)-g(y)|\right] \ln \frac{1}{x}<\infty .
$$

This condition is weaker than known logarithmic condition (3); that is, (3) implies (4). We can give an example of exponential function $p(\cdot)$ for which condition $(4)$ is satisfied but (3) fails: $p(x)=p(0)+\left(\epsilon /(\ln (1 / x))^{\alpha}\right) ; 0<\alpha<1, \epsilon>0$. Also using L'Hopital's rule, it is not difficult to check that such a function $p$ satisfies the condition (5). Therefore, applying the assertion of our results below, we get new results on existence of inequality (1) (compare with the known results in [17] or [24]; the condition (3) was imposed there).

The following main result is obtained in this paper.

Theorem 1. Let $\beta:(0, l) \rightarrow \mathbb{R}$ and $p:(0, l) \rightarrow(1, \infty)$ be measurable functions such that $\beta(0)<1 / p^{\prime}(0)$. Suppose there are limits $\lim _{x \rightarrow+0} p(x)=p(0) \in(1, \infty)$ and $\lim _{x \rightarrow+0} \beta(x)=$ $\beta(0) \in \mathbb{R}$ and the functions $\beta, p$ satisfy condition (4) near origin.

Then inequality (1) holds if and only if

$$
\int_{a}^{l} x^{\beta(x)-1 / p^{\prime}(x)} \frac{d x}{x} \leq C a^{\beta(a)-1 / p^{\prime}(a)}, \quad a \in(0, l) .
$$

\section{Notation}

As to the basic properties of spaces $L^{p(\cdot)}$, we refer to $[26,27]$. Throughout this paper, it is assumed that $p(x)$ is a measurable function in $(0, l)$ taking its values from the interval $[1, \infty)$ with $p^{+}=\sup \{p(x): x \in(0, l)\}<\infty$. The space of functions $L^{p(\cdot)}(0, l)$ is introduced as the class of measurable functions $f(x)$ in $(0, l)$ which has a finite $I_{p(\cdot)}(f)=\int_{0}^{l}|f(x)|^{p(x)} d x$ modular (put also $I_{p(\cdot) ; E}(f)=\int_{E}|f(t)|^{p(t)} d t$ ). A norm in $L^{p(\cdot)}(0, l)$ is given in the form

$$
\|f\|_{p(\cdot)}=\inf \left\{\lambda>0: I_{p(\cdot)}\left(\frac{f}{\lambda}\right) \leq 1\right\}
$$

(By $\|f\|_{p(\cdot) ; E}$ or simply $\|f\|_{p(\cdot)}$, we denote the $p(\cdot)$ norm over set $E$.)

For $1<p^{-}, p^{+}<\infty$, the space $L^{p(\cdot)}(0, l)$ is a reflexive Banach space. The relation between the modular and norm is expressed by the following inequalities (see, e.g., [12]):

$$
\begin{gathered}
\|f\|_{p(\cdot)}^{p^{+}} \leq I_{p}(f) \leq\|f\|_{p(\cdot)}^{p^{-}}, \quad\|f\|_{p(\cdot),} \leq 1, \\
\|f\|_{p(\cdot)}^{p^{-}} \leq I_{p}(f) \leq\|f\|_{p(\cdot)}^{p^{+}}, \quad\|f\|_{p(\cdot)}>1 .
\end{gathered}
$$

These inequalities allow us to perform our norm estimates in terms of a modular.

For the function $1 \leq p(x)<\infty, p^{\prime}(x)$ denotes the conjugate function of $p(x),(1 / p(x))+\left(1 / p^{\prime}(x)\right)=1$, and $p^{\prime}(x)=$ $\infty$ if $p(x)=1$. We denote by $C, C_{1}, C_{2}, \ldots$ various positive constants whose values may vary at each appearance. We write $u \sim v$ if there exist positive constants $C_{1}, C_{2}$ such that
$C_{1} u(x) \leq v(x) \leq C_{2} u(x)$. By $\chi_{E}$, we denote the characteristic function of set $E$.

We say a function $u:(0, l) \rightarrow(-\infty,+\infty)$ is almost increasing (decreasing) if there exists a constant $C>0$ such that $u\left(t_{1}\right) \leq \mathrm{Cu}\left(t_{2}\right)$ and $\left(u\left(t_{2}\right) \leq \mathrm{Cu}\left(t_{1}\right)\right)$ for $0<t_{1} \leq t_{2}<l$.

\section{Proofs}

Throughout the section, we assume that $p:(0, l) \rightarrow[1, \infty)$ and $\beta:(0, l) \rightarrow(-\infty+\infty)$ are measurable functions such that $p^{+}<\infty,-\infty<\beta^{-}$, and $\beta^{+}<\infty$.

Lemma 2. Suppose $s:(0, l) \rightarrow \mathbb{R}$ is a measurable function such that $-\infty<s^{-}, s^{+}<+\infty$. and the condition (4) be satisfied. Then there exists a constant $C>1$ such that

$$
\frac{1}{C} y^{-s(y)} \leq x^{-s(x)} \leq C y^{-s(y)}
$$

for any $0<x<l, y \in(x / 2,3 x / 2)$, where the constant $C$ depends on $s^{-}, s^{+}$, and the constant from the condition (4).

Proof. Since $s$ satisfies the condition (4), it follows that, if $s(y) \geq 0$, then

$$
\begin{aligned}
y^{-s(y)} & \leq\left(\frac{x}{2}\right)^{-s(y)+s(x)}\left(\frac{x}{2}\right)^{-s(x)} \\
& \leq e^{(s(x)-s(y)) \ln (2 / x)} 2^{s(x)} x^{-s(x)} \leq C x^{-s(x)},
\end{aligned}
$$

and if $s(y)<0$ then $y^{-s(y)} \leq((3 / 2) x)^{-s(y)} \leq C x^{-s(x)}$. By the same arguments,

$$
x^{-s(x)} \leq C y^{-s(y)}
$$

Proof of Theorem 1. Consider the following.

Necessity. First, show that the function $x^{\beta(x)-\left(1 / p^{\prime}(x)\right)}$ is almost decreasing if inequality (1) holds and the functions $p, \beta$ satisfy (4). In this way, we will show that

$$
t^{\beta(t)-1 / p^{\prime}(t)} \leq C_{1} a^{\beta(a)-1 / p^{\prime}(a)}, \quad 0<a<t<l<\infty,
$$

where the constant $C$ does not depend on $a, t$. For fixed $t, a$, there exists an $m \in \mathbb{N}$ such that

$$
2^{m-1} a<t \leq 2^{m} a \text {. }
$$

Let $n_{0} \in \mathbf{N}, n_{0} \geq m$, be such that $2^{n_{0}} a \leq l<2^{n_{0}+1} a$. Insert a test function

$$
f_{0}(x)=x^{-\beta(x)-1 / p(x)} \chi_{(a / 2, a)}(x), \quad x \in(0, l),
$$

into inequality (1). Then

$$
I_{p(\cdot)}\left(x^{\beta(x)} f_{0}\right)=\int_{a / 2}^{a} x^{-1} d t=\ln 2,
$$


and, therefore, $\left\|x^{\beta} f_{0}\right\|_{p(\cdot)} \leq 1$. It follows from (1) that $\left\|x^{\beta-1} H f_{0}\right\|_{p(\cdot)} \leq C$. This means that $I_{p(\cdot)}\left(x^{\beta(x)-1} H f_{0}\right) \leq C_{2}$. Therefore,

$$
\int_{a}^{l}\left[x^{\beta(x)-1 / p^{\prime}(x)} \int_{a / 2}^{a} u^{-\beta(u)+1 / p^{\prime}(u)} \frac{d u}{u}\right]^{p(x)} \frac{d x}{x} \leq C_{2} .
$$

Hence,

$$
\begin{aligned}
& \sum_{n=1}^{n_{0}} \int_{2^{n-1} a}^{2^{n} a}\left[x^{\beta(x)-1 / p^{\prime}(x)} \int_{a / 2}^{a} u^{-\beta(u)+1 / p^{\prime}(u)} \frac{d u}{u}\right]^{p(x)} \frac{d x}{x} \leq C_{2}, \\
& \int_{2^{m-1} a}^{2^{m} a}\left[x^{\beta(x)-1 / p^{\prime}(x)} \int_{a / 2}^{a} u^{-\beta(u)+1 / p^{\prime}(u)} \frac{d u}{u}\right]^{p(x)} \frac{d x}{x} \leq C_{2} .
\end{aligned}
$$

In the integral term, it easily follows from Lemma 2 and (4) for $p, \beta$ that

$$
C_{3} u^{-\beta(u)+1 / p^{\prime}(u)} \geq a^{-\beta(a)+1 / p^{\prime}(a)}, \quad u \in(a / 2, a) .
$$

Using this estimate and (17), (15), we see that

$$
\begin{gathered}
\int_{2^{m-1} a}^{2^{m} a}\left[\frac{\ln 2}{C_{3}} a^{1 / p^{\prime}(a)-\beta(a)} x^{\beta(x)-1 / p^{\prime}(x)}\right]^{p(x)} \frac{d x}{x} \leq C_{2}, \\
\int_{a}^{l}\left[x^{\beta(x)-1 / p^{\prime}(x)} a^{-\beta(a)+1 / p^{\prime}(a)}\right]^{p(x)} \frac{d x}{x} \leq C_{4},
\end{gathered}
$$

respectively.

Using the Holder inequality for $p(\cdot)$-norms, we see that

$$
\begin{aligned}
& \int_{2^{m-1} a}^{2^{m} a}\left[a^{1 / p^{\prime}(a)-\beta(a)} x^{\beta(x)-1 / p^{\prime}(x)}\right] \frac{d x}{x} \\
& \leq C_{0}\left\|\chi_{\left(2^{m-1} a, 2^{m} a\right)} x^{-1 / p^{\prime}(\cdot)}\right\|_{p^{\prime}(\cdot)} \\
& \quad \times\left\|\chi_{\left(2^{m-1} a, 2^{m} a\right)} x^{-1 / p(\cdot)} C a^{1 / p^{\prime}(a)-\beta(a)} x^{\beta(x)-1 / p^{\prime}(x)}\right\|_{p(\cdot)} \\
& \leq C_{0} C
\end{aligned}
$$

since

$$
I_{p^{\prime}(\cdot)}\left(\chi_{\left(2^{m-1} a, 2^{m} a\right)} x^{-1 / p^{\prime}(\cdot)}\right)=\int_{2^{m-1} a}^{2^{m} a} \frac{d x}{x}=\ln 2,
$$

and by (19)

$$
\begin{aligned}
& I_{p(\cdot)}\left(x^{-1 / p(\cdot)} C a^{1 / p^{\prime}(a)-\beta(a)} x^{\beta(x)-1 / p^{\prime}(x)} \cdot \chi_{\left(2^{m-1} a, 2^{m} a\right)}(x)\right) \\
& \quad \leq C_{5} .
\end{aligned}
$$

Hence

$$
\int_{2^{m-1} a}^{2^{m} a}\left[a^{1 / p^{\prime}(a)-\beta(a)} x^{\beta(x)-1 / p^{\prime}(x)}\right] \frac{d x}{x} \leq C,
$$

and then

$$
\int_{2^{m-1} a}^{2^{m} a} x^{\beta(x)-1 / p^{\prime}(x)} \frac{d x}{x} \leq C a^{\beta(a)-1 / p^{\prime}(a)} .
$$

On the other hand, by Lemma 2 and (4) for $p, \beta$, we have

$$
x^{\beta(x)-1 / p^{\prime}(x)} \geq C t^{\beta(t)-1 / p^{\prime}(t)}, \quad x \in\left(2^{m-1} a, 2^{m} a\right) .
$$

Using this inequality from (25), we get (11). Inequity (11) has been proved; that is, the function $x^{\beta(x)-\left(1 / p^{\prime}(x)\right)}$ is almost decreasing.

Now, it follows from (11) and (20) that

$$
\begin{aligned}
C & \geq \int_{a}^{l}\left[\frac{x^{\beta(x)-1 / p^{\prime}(x)} a^{-\beta(a)+1 / p^{\prime}(a)}}{C_{1}}\right]^{p(x)} C_{1}^{p(x)} \frac{d x}{x} \\
& \geq \int_{a}^{l}\left[\frac{x^{\beta(x)-1 / p^{\prime}(x)} a^{-\beta(a)+1 / p^{\prime}(a)}}{C_{1}}\right]^{p^{+}} C_{1} \frac{d x}{x} .
\end{aligned}
$$

Hence,

$$
\int_{a}^{l} x^{\left(\beta(x)-1 / p^{\prime}(x)\right) p^{+}} \frac{d x}{x} \leq C a^{\left(\beta(a)-1 / p^{\prime}(a)\right) p^{+}} .
$$

Now, using (28), we will derive a Bari-Stechkin [28] type assertion in order to prove that the function $x^{\left(\beta(x)-1 / p^{\prime}(x)\right) p^{+}+\varepsilon}$ is almost decreasing by some $\varepsilon>0$.

Indeed, put $g(x)=\int_{x}^{l} t^{\left(\beta(t)-1 / p^{\prime}(t)\right) p^{+}}(d t / t)$. Then, by (28),

$$
-x g^{\prime}(x) \geq \frac{1}{C} g(x), \quad 0<x<l .
$$

Integrating this inequality,

$$
x_{1}^{1 / C} g\left(x_{1}\right) \geq x_{2}^{1 / C} g\left(x_{2}\right), \quad 0<x_{1} \leq x_{2} \leq \frac{1}{2} l .
$$

Using (28),

$$
C x_{1}^{1 / C+p^{+}\left(\beta\left(x_{1}\right)-1 / p^{\prime}\left(x_{1}\right)\right)} \geq x_{2}^{1 / C} g\left(x_{2}\right) .
$$

Now, it follows from Lemma 2 and (4) for $p, \beta$ that

$$
g\left(x_{2}\right) \geq \int_{x_{2}}^{2 x_{2}} x^{\left(\beta(x)-1 / p^{\prime}(x)\right) p^{+}} \frac{d x}{x} \geq C x_{2}^{\left(\beta\left(x_{2}\right)-1 / p^{\prime}\left(x_{2}\right)\right) p^{+}} .
$$

Therefore,

$$
C x_{1}^{1 / C+\left(\beta\left(x_{1}\right)-1 / p^{\prime}\left(x_{1}\right)\right) p^{+}} \geq x_{2}^{1 / C+\left(\beta\left(x_{2}\right)-1 / p^{\prime}\left(x_{2}\right)\right) p^{+}} ;
$$

that is, the function $x^{\varepsilon+\left(\beta(x)-\left(1 / p^{\prime}(x)\right)\right) p^{+}}$is almost decreasing by $\varepsilon=1 / C$. This implies almost decreasing of the function $x^{\beta(x)-1 / p^{\prime}(x)+\varepsilon_{1}}$ by $\varepsilon_{1}=\varepsilon / p^{+}$. Then it is easily seen that the condition (5) is satisfied.

This completes necessity of condition (5).

Sufficiency. Let the functions $p, \beta$ satisfy (4) and the condition (5). Show that (5) implies almost decreasing of $x^{\beta(x)-1 / p^{\prime}(x)+\varepsilon}$ by some $\varepsilon>0$. Put $g(x)=\int_{x}^{l} t^{\beta(t)-1 / p^{\prime}(t)} d t / t$ and repeat the arguments before. 
We have

$$
\begin{gathered}
-x g^{\prime}(x) \geq \frac{1}{C} g(x), \quad \text { integrating, } \\
x_{1}^{1 / C} g\left(x_{1}\right) \geq x_{2}^{1 / C} g\left(x_{2}\right), \quad 0<x_{1} \leq x_{2} \leq \frac{1}{2} l .
\end{gathered}
$$

Using (5),

$$
C x_{1}^{1 / C+\beta(x)-1 / p^{\prime}\left(x_{1}\right)} \geq x_{2}^{1 / C} g\left(x_{2}\right) \text {. }
$$

By Lemma 2 and (4) for $p, \beta$, it follows that

$$
g\left(x_{2}\right) \geq \int_{x_{2}}^{2 x_{2}} x^{\beta(x)-1 / p^{\prime}(x)} \frac{d x}{x} \geq C x_{2}^{\beta\left(x_{2}\right)-1 / p^{\prime}\left(x_{2}\right)} .
$$

Hence,

$$
x_{2}^{1 / C+\beta\left(x_{2}\right)-1 / p^{\prime}\left(x_{2}\right)} \leq C x_{1}^{1 / C+\beta\left(x_{1}\right)-1 / p^{\prime}\left(x_{1}\right)}
$$

that is, $x^{\varepsilon+\beta(x)-1 / p^{\prime}(x)}$ is almost decreasing by $\varepsilon=1 / C$.

Let $f(x) \geq 0$ be a measurable function such that $\left\|x^{\beta(x)} f\right\|_{p(\cdot)} \leq 1$. Then $I_{p(\cdot)}\left(x^{\beta(x)} f\right) \leq 1$. We have to prove $\left\|x^{\beta(x)-1} H f\right\|_{p(\cdot)} \leq C_{1}$. By Minkowskii inequality for $L^{p(\cdot)}$ norms,

$$
\begin{aligned}
\left\|x^{\beta(x)-1} H f\right\|_{p(\cdot)} \leq & \left\|x^{\beta(x)-1} \int_{0}^{x} f(t) d t\right\|_{p(\cdot) ;(\delta, l)} \\
& +\sum_{n=0}^{\infty}\left\|x^{\beta(x)-1} \int_{2^{-n-1} x}^{2^{-n} x} f(t) d t\right\|_{p(\cdot) ;(0, \delta)},
\end{aligned}
$$

where $0<\delta<(1 / 2) l$ is a fixed number.

We will derive an estimate for every summand in (38). In this way, we will get a modular estimate for the corresponding terms in modular.

Denote

$$
p_{x, n}^{-}=\inf \left\{p(t): t \in\left(2^{-n-1} x, 2^{-n} x\right)\right\} ; \quad n=1,2, \ldots
$$

By (37),

$$
x^{\beta(x)-1 / p^{\prime}(x)+\varepsilon} \leq C t^{\beta(t)-1 / p^{\prime}(t)+\varepsilon}
$$

for any $t \in\left(2^{-n-1} x, 2^{-n} x\right), 0<x<\delta$, where $C$ does not depend on $n, t, x$. From (40) using $2^{-n-1} x<t<2^{-n} x$, we get

$$
\begin{aligned}
t^{1 / p^{\prime}(t)-\beta(t)} & =t^{\epsilon} t^{1 / p^{\prime}(t)-\beta(t)-\epsilon} \leq C t^{\epsilon} x^{1 / p^{\prime}(x)-\beta(x)-\epsilon} \\
& \leq C 2^{-n \epsilon} x^{1 / p^{\prime}(x)-\beta(x)}
\end{aligned}
$$

or

$$
x^{-1 / p^{\prime}(x)+\beta(x)} \leq C 2^{-n \epsilon} t^{-1 / p^{\prime}(t)+\beta(t)} .
$$

If $p_{x, n}^{-} \leq p(x)$, then, due to Holder's inequality, for $x \in$ $(0, \delta)$ we have

$$
\begin{aligned}
& x^{\beta(x)-1} \int_{2^{-n-1} x}^{2^{-n} x} t^{\beta(t) p^{\prime}(t)} f(t) t^{-\beta(t) p^{\prime}(t)} d t \\
& \leq x^{\beta(x)-1}\left(\int_{2^{-n-1} x}^{2^{-n} x} t^{1-\beta(t) p^{\prime}(t)} \frac{d t}{t}\right)^{1 /\left(p_{x, n}^{-}\right)^{\prime}} \\
& \quad \times\left(\int_{2^{-n-1} x}^{2^{-n} x}\left(t^{\beta(t)} f(t)\right)^{p_{x, n}^{-}} \cdot t^{-\beta(t) p^{\prime}(t)} d t\right)^{1 / p_{x, n}^{-}} .
\end{aligned}
$$

Using (4) for $p, \beta$ and Lemma 2, it is not difficult to see the following estimate:

$$
\left(\int_{2^{-n-1} x}^{2^{-n} x} s^{1-\beta(s) p^{\prime}(s)} \frac{d s}{s}\right)^{1 /\left(p_{x, n}^{-}\right)^{\prime}} \leq C t^{1 / p^{\prime}(t)-\beta(t)},
$$

where $t \in\left(2^{-n-1} x, 2^{-n} x\right)$ and $0<x<\delta, n=1,2, \ldots$, with the constant $C>0$ not depending on $n, x, t$.

Indeed, $s^{1-\beta(s) p^{\prime}(s)} \sim t^{1-\beta(t) p^{\prime}(t)}$ for $p, \beta$ and $s, t \in$ $\left(2^{-n-1} x, 2^{-n} x\right)$. Then to prove (44), it suffices to show $t^{1 /\left(p_{x, n}^{-}\right)^{\prime}} \sim t^{1 / p^{\prime}(t)}$, which is a simple consequence of (4) for $p$, Lemma 2, and the fact that there exists a point $y \in$ $\left(2^{-n-1} x, 2^{-n} x\right)$ such that $\left|p_{x, n}^{-}-p(y)\right| \leq C /|\ln t|$.

For the second multilayer (43), we have the estimates

$$
\begin{aligned}
& \int_{2^{-n-1} x}^{2^{-n} x}\left(t^{\beta(t) p^{\prime}(t)} f(t)\right)^{p_{x, n}^{-}} t^{-\beta(t) p^{\prime}(t)} d t \\
& \leq \int_{2^{-n-1} x}^{2^{-n} x} \chi_{\left\{t^{\beta(\cdot)} p^{\prime}(\cdot) f \geq 1\right\}}\left(t^{\beta(t) p^{\prime}(t)} f(t)\right)^{p(t)} t^{-\beta(t) p^{\prime}(t)} d t \\
& \quad+\int_{2^{-n-1} x}^{2^{-n} x} t^{-\beta(t) p^{\prime}(t)} d t \\
& \leq \int_{2^{-n-1} x}^{2^{-n} x}\left(\left(t^{\beta(t) p^{\prime}(t)} f(t)\right)^{p(t)}+1\right) t^{-\beta(t) p^{\prime}(t)} d t .
\end{aligned}
$$

Combining the estimates (43), (44), and (45) for $0<x<\delta$ and $t \in\left(2^{-n-1} x<t \leq 2^{-n} x\right)$ we have

$$
\begin{aligned}
x^{\beta(x)-1} & \int_{2^{-n-1} x}^{2^{-n} x} f(t) d t \\
\leq & C x^{-1 / p(x)}\left(t^{1 / p^{\prime}(t)-\beta(t)} x^{\beta(x)-1 / p^{\prime}(x)}\right) \\
& \times\left\{\int_{2^{-n-1} x}^{2^{-n} x}\left(\left(t^{\beta(t)} f(t)\right)^{p(t)}+t^{-\beta(t) p^{\prime}(t)}\right) d t\right\}^{1 / p_{x, n}^{-}} .
\end{aligned}
$$

Now, taking into the account (42), here, we see that the last term is exceeded by

$$
\begin{aligned}
& \leq C 2^{-n \varepsilon} x^{-(1 / p(x))} \\
& \times\left[\left\{\int_{2^{-n-1} x}^{2^{-n} x}\left(\left(t^{\beta(t)} f(t)\right)^{p(t)}+t^{-\beta(t)(t) p^{\prime}(t)}\right) d t\right\}^{1 / p(x)}\right]^{p(x) / p_{x, n}^{-}} .
\end{aligned}
$$


On the other hand, using the assumptions $\beta(0)<1 / p^{\prime}(0)$ and $I_{p}\left(x^{\beta} f\right) \leq 1$,

$$
\begin{aligned}
& \int_{2^{-n-1} x}^{2^{-n} x}\left(\left(t^{\beta(t)} f(t)\right)^{p(t)}+t^{-\beta(t) p^{\prime}(t)}\right) d t \\
& \quad \leq 1+C t^{1-\beta(t) p^{\prime}(t)} \leq C_{1}, \quad t \in\left(2^{-n-1} x<t \leq 2^{-n} x\right) .
\end{aligned}
$$

Using (48) and $p_{x, n}^{-} \leq p(x)$, it follows from (47) that

$$
\begin{aligned}
& x^{\beta(x)-1} \int_{2^{-n-1} x}^{2^{-n} x} f(t) d t \\
& \leq C C_{1}^{p^{+} / p^{-}-1} 2^{-n \varepsilon} x^{-1 / p(x)}\left\{\int _ { 2 ^ { - n - 1 } x } ^ { 2 ^ { - n } x } \left(\left(t^{\beta(t)} f(t)\right)^{p(t)}\right.\right. \\
& \\
& \left.\left.+t^{-\beta(t)(t) p^{\prime}(t)}\right) d t\right\}^{1 / p(x)} .
\end{aligned}
$$

If $p_{x, n}^{-}>p(x)$, then we repeat all arguments with $p_{x, n}^{-}$changed to $p(x)$. Indeed, it follows from the Holder inequality that

$$
\begin{aligned}
x^{\beta(x)-1} & \int_{2^{-n-1} x}^{2^{-n} x} f(t) d t \\
\leq & x^{\beta(x)-1}\left(\int_{2^{-n-1} x}^{2^{-n} x}\left(t^{\beta(t) p^{\prime}(t)} f(t)\right)^{p(x)} t^{-\beta(t) p^{\prime}(t)} d t\right)^{1 / p(x)} \\
& \times\left(\int_{2^{-n-1} x}^{2^{-n} x} t^{1-\beta(t) p^{\prime}(t)} \frac{d t}{t}\right)^{1 / p^{\prime}(x)} .
\end{aligned}
$$

By (4), for $p, \beta$, we have (see similar arguments after (44))

$$
\begin{array}{r}
\left(\int_{2^{-n-1} x}^{2^{-n} x} s^{1-\beta(s) p^{\prime}(s)} \frac{d s}{s}\right)^{1 / p^{\prime}(t)} \leq C t^{\left(1 / p^{\prime}(t)\right)-\beta(t)}, \\
t \in\left(2^{-n-1} x<t \leq 2^{-n} x\right) .
\end{array}
$$

Also,

$$
\begin{aligned}
& \int_{2^{-n-1} x}^{2^{-n} x}\left(t^{\beta(t) p^{\prime}(t)} f(t)\right)^{p(x)} t^{-\beta(t) p^{\prime}(t)} d t \\
& \leq \int_{2^{-n-1} x}^{2^{-n} x} \chi_{\left\{t^{\beta(\cdot) p^{\prime}(\cdot)} f \geq 1\right\}}\left(t^{\beta(t) p^{\prime}(t)} f(t)\right)^{p(t)} t^{-\beta(t) p^{\prime}(t)} d t \\
& \quad+\int_{2^{-n-1} x}^{2^{-n} x} t^{-\beta(t) p^{\prime}(t)} d t \\
& \leq 1+C t^{1-\beta(t) p^{\prime}(t)} \leq C_{1}, \quad t \in\left(2^{-n-1} x<t \leq 2^{-n} x\right)
\end{aligned}
$$

since the conditions $\beta(0) p^{\prime}(0)<1, p(x)<p_{x, n}^{-}$, and $I_{p(\cdot) ;(0,1)}\left(x^{\beta} f\right) \leq 1$ are assumed.
Hence,

$$
\int_{2^{-n-1} x}^{2^{-n} x}\left(t^{\beta(t) p^{\prime}(t)} f(t)\right)^{p(x)} t^{-\beta(t) p^{\prime}(t)} d t \leq C_{1} .
$$

Therefore, by use of (42) for $0<x<\delta$ and $t \in\left(2^{-n-1} x, 2^{-n}\right)$, it follows that

$$
\begin{aligned}
x^{\beta(x)-1} & \int_{2^{-n-1} x}^{2^{-n} x} f(t) d t \\
\leq & C\left[t^{\left(1 / p^{\prime}(t)-\beta(t)\right) p^{\prime}(t)} x^{\beta(x)-1 / p^{\prime}(x)}\right]^{1 / p^{\prime}(x)} x^{-(1 / p(x))} \\
& \times\left\{\int_{2^{-n-1} x}^{2^{-n} x}\left(\left(t^{\beta(t)} f(t)\right)^{p(t)}+t^{-\beta(t) p^{\prime}(t)}\right) d t\right\}^{1 / p(x)} \\
\leq & C 2^{-n \varepsilon / p^{\prime}(x)} t^{\left(1 / p^{\prime}(t)-\beta(t)\right)\left(\left(p^{\prime}(t)-1\right) / p^{\prime}(x)\right)} x^{-(1 / p(x))} \\
& \times\left\{\int_{2^{-n-1} x}^{2^{-n} x}\left(\left(t^{\beta(t)} f(t)\right)^{p(t)} d t+t^{-\beta(t) p^{\prime}(t)}\right) d t\right\}^{1 / p(x)} .
\end{aligned}
$$

Let us note that

$$
t^{\left(1 / p^{\prime}(t)-\beta(t)\right)\left(p^{\prime}(t)-1\right) / p^{\prime}(x)} \leq C, \quad 2^{-n-1} x<t<2^{-n} x
$$

since $\beta(0)<1 / p^{\prime}(0)$. Therefore,

$$
\begin{gathered}
x^{\beta(x)-1} \int_{2^{-n-1} x}^{2^{-n} x} f(t) d t \\
\leq C_{1} 2^{-n \varepsilon_{1}} x^{-1 / p(x)}\left\{\int _ { 2 ^ { - n - 1 } x } ^ { 2 ^ { - n } x } \left(\left(t^{\beta(t)} f(t)\right)^{p(t)}\right.\right. \\
\left.\left.+t^{-\beta(t) p^{\prime}(t)}\right) d t\right\}^{1 / p(x)},
\end{gathered}
$$

with $\varepsilon_{1}=\varepsilon / p^{\prime}(0)$.

It follows from (56) and (49) that, in both cases $p(x)<$ $p_{x, n}^{-}$and $p(x) \geq p_{x, n}^{-}$, we have the same estimates (with different $C, \varepsilon$ not depending on $x, n$.). Denote again by $\varepsilon$ the minimum of $\varepsilon$ and $\varepsilon_{1}$ and, taking into account (56) and (49), we get

$$
\begin{aligned}
& I_{p(\cdot) ;(0, \delta)}\left(x^{\beta(x)-1} \int_{2^{-n-1} x}^{2^{-n} x} f(t) d t\right) \\
& \leq C C_{1}^{p^{+} / p^{-}-1} 2^{-n \varepsilon p^{-}} \\
& \quad \times \int_{0}^{\delta} x^{-1}\left(\int_{2^{-n-1} x}^{2^{-n} x}\left(\left(t^{\beta(t)} f(t)\right)^{p(t)}+t^{-\beta(t) p^{\prime}(t)}\right) d t\right) d x .
\end{aligned}
$$


Due to Fubini's theorem, this is exceeded by

$$
\begin{aligned}
& C_{1}^{p^{+} / p^{-}-1} 2^{-n \varepsilon p^{-}} \int_{0}^{\delta 2^{-n}}\left(\int_{2^{n-1} t}^{2^{n} t} x^{-1} d x\right) \\
& \quad \times\left(\left(t^{\beta(t)} f(t)\right)^{p(t)}+t^{-\beta(t) p^{\prime}(t)}\right) d t \\
& =C_{1}^{p^{+} / p^{-}-1} 2^{-n \varepsilon p^{-}} \ln 2 \int_{0}^{\delta 2^{-n}}\left(\left(t^{\beta} f(t)\right)^{p(t)}+t^{-\beta(t) p^{\prime}(t)}\right) d t \\
& \leq C_{6} 2^{-n \varepsilon p^{-}} .
\end{aligned}
$$

We have used that $I_{p(\cdot)}\left(t^{\beta(x)} f(\cdot)\right) \leq 1$ and the estimate

$$
\int_{0}^{\delta 2^{-n}} t^{-\beta(t) p^{\prime}(t)} d t \leq C
$$

which easily follows from $\beta(0)<1 / p^{\prime}(0)$.

Therefore,

$$
\left\|x^{\beta(x)-1} \int_{2^{-n-1} x}^{2^{-n} x} f(t) d t\right\|_{L^{p(\cdot)}(0, \delta)} \leq C 2^{-n \varepsilon p^{-} / p^{+}} .
$$

From this estimate and (38), it follows that

$$
\left\|x^{\beta(x)-1} H f\right\|_{p(\cdot) ;(0, \delta)} \leq C \sum_{n=0}^{\infty} 2^{-n \varepsilon p^{-} / p^{+}} \leq C_{1} .
$$

It remains to get an estimate of $\left\|x^{\beta(x)-1} \int_{0}^{x} f(t) d t\right\|_{p(\cdot) ;(\delta, l)}$ far from origin. Since $x^{\beta(x)-1}$ is separated from zero and infinity in $(\delta, l)$, it suffices to note the estimate

$$
\int_{0}^{x} f(t) d t \leq\left\|t^{\beta(t)} f\right\|_{p(\cdot) ;(0, l)}\left\|t^{-\beta(t)}\right\|_{p^{\prime}(\cdot) ;(0, l)} \leq C .
$$

Here, the boundedness of first multiplier follows from the assumption. Boundedness of second multiplier follows from the condition $\beta(0)<1 / p^{\prime}(0)$ and the assertion of Lemma 2.

Theorem 1 has been proved.

\section{Conflict of Interests}

The authors declare that there is no conflict of interests regarding the publication of this paper.

\section{Acknowledgments}

The authors are grateful to the reviewer's valuable comments that improved the paper. The first author is grateful to the Science Development Foundation under the President of Azerbaijan Republic (EIF-2012-2(6)-39/09/1). The authors are also grateful to the Scientific and Technological Research Council of Turkey for a scholarship (TÜBİTAK-BİDEB, 2012).

\section{References}

[1] H. Triebel, Interpolation Theory, Function Spaces, Differential Operators, North-Holland Publishing, 1978.

[2] A. Kufner, L. Maligranda, and L.-E. Persson, The Hardy Inequality: About Its History and Some Related Results, Vydavatelský servis, 2007.

[3] J. L. Lions and E. Magenes, Non Homogeneous Boundary Problem and Applications, vol. 2, Springer, New York, NY, USA, 1972.

[4] Y. Brundyi and N. Krugljak, Interpolation Functors and Interpolation Spaces, North-Holland Publisher, 1991.

[5] M. Ruzicka, Electrorheological Fluids Modeling and Mathematical the Ory, Lecture Notes in Mathematics, Springer, Berlin, Germany, 2000.

[6] V. V. Zhikov, "On some variational problems," Russian Journal of Mathematical Physics, vol. 5, no. 1, pp. 105-116, 1997.

[7] P. Marcellini, "Regularity for elliptic equations with general growth conditions," Journal of Differential Equations, vol. 105, no. 2, pp. 296-333, 1993.

[8] E. Acerbi and G. Mingione, "Regularity results for a class of functionals with non-standard growth," Archive for Rational Mechanics and Analysis, vol. 156, no. 2, pp. 121-140, 2001.

[9] L. Diening, P. Harjulehto, P. Hästö, and M. Ruzicka, Lebesgue and Sobolev Spaces with Variable Exponents, vol. 2017 of Lecture Notes in Mathematics, Springer, Heidelberg, Germany, 2011.

[10] D. Cruz-Uribe and A. Fiorenza, Variable Lebesgue Spaces, Fondation and Harmonic Analysis, Birkhäuser, 2013.

[11] S. Samko, "Hardy inequality in the generalized Lebesgue spaces," Fractional Calculus \& Applied Analysis, vol. 6, no. 4, pp. 355-362, 2003.

[12] S. G. Samko, "Convolution type operators in $L^{p(x)}$," Integral Transforms and Special Functions, vol. 7, no. 1-2, pp. 123-144, 1998.

[13] V. Kokilashvili and S. Samko, "Maximal and fractional operators in weighted $L^{p(x)}$ spaces," Revista Matemática Iberoamericana, vol. 20, no. 2, pp. 493-515, 2004.

[14] P. Harjulehto, P. Hästö, and M. Koskenoja, "Hardy’s inequality in a variable exponent Sobolev space," Georgian Mathematical Journal, vol. 12, no. 3, pp. 431-442, 2005.

[15] D. E. Edmunds, V. Kokilashvili, and A. Meskhi, "On the boundedness and compactness of weighted Hardy operators in spaces $L^{p(x)}$, , Georgian Mathematical Journal, vol. 12, no. 1, pp. 27-44, 2005.

[16] H. Rafeiro and S. Samko, "Hardy type inequality in variable Lebesgue spaces," Annales Academice Scientiarum Fennica. Mathematica, vol. 34, no. 1, pp. 279-289, 2009.

[17] L. Diening and S. Samko, "Hardy inequality in variable exponent Lebesgue spaces," Fractional Calculus \& Applied Analysis, vol. 10, no. 1, pp. 1-17, 2007.

[18] R. A. Mashiyev, B. Çekiç, F. I. Mamedov, and S. Ogras, "Hardy's inequality in power-type weighted $L^{p(\cdot)}(0, \infty)$ spaces," Journal of Mathematical Analysis and Applications, vol. 334, no. 1, pp. 289-298, 2007.

[19] F. I. Mamedov and A. Harman, "On a weighted inequality of Hardy type in spaces $L^{p(\cdot)}$," Journal of Mathematical Analysis and Applications, vol. 353, no. 2, pp. 521-530, 2009.

[20] F. I. Mamedov and A. Harman, "On a Hardy type general wei-

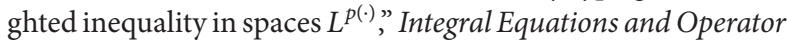
Theory, vol. 66, no. 4, pp. 565-592, 2010.

[21] D. Cruz-Uribe and F. I. Mamedov, "On a general weighted Hardy type inequality in the variable exponent Lebesgue 
spaces," Revista Matemática Complutense, vol. 25, no. 2, pp. 335367, 2012.

[22] F. I. Mamedov and Y. Zeren, "On equivalent conditions for the

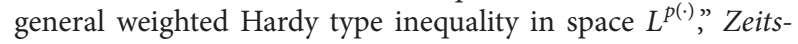
chrift für Analysis und ihre Anwendungen, vol. 31, no. 1, pp. 5574, 2012.

[23] F. I. Mamedov, "On Hardy type inequality in variable exponent Lebesgue space $L^{p(\cdot)}(0, l)$," Azerbaijan Journal of Mathematics, vol. 2, no. 1, pp. 90-99, 2012.

[24] A. Harman and F. I. Mamedov, "On boundedness of weighted Hardy operator in $L^{p(\cdot)}$ and regularity condition," Journal of Inequalities and Applications, vol. 2010, Article ID 837951, 14 pages, 2010.

[25] F. I. Mamedov and F. M. Mammadova, "A necessary and suffic-

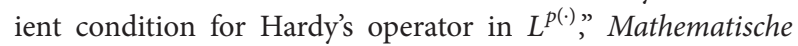
Nachrichten, vol. 287, no. 5-6, pp. 666-676, 2013.

[26] X. Fan and D. Zhao, "On the spaces $L^{p(x)}(\Omega)$ and $W^{m, p(x)}(\Omega)$," Journal of Mathematical Analysis and Applications, vol. 263, no. 2, pp. 424-446, 2001.

[27] O. Kováčik and J. Rákosník, "On spaces $L^{p(x)}(\Omega)$ and $W^{1, p(x), "}$ Czechoslovak Mathematical Journal, vol. 41, no. 116, pp. 592-618, 1991.

[28] N. K. Bari and S. B. Stečkin, "Best approximations and differential properties of two conjugate functions," Proceedings of Moscow Mathematical Society, vol. 5, pp. 483-522, 1956. 


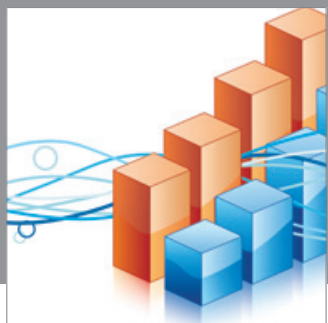

Advances in

Operations Research

mansans

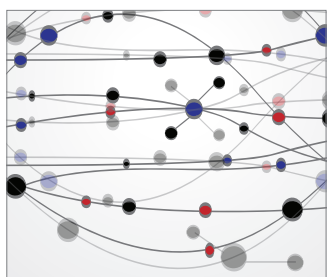

The Scientific World Journal
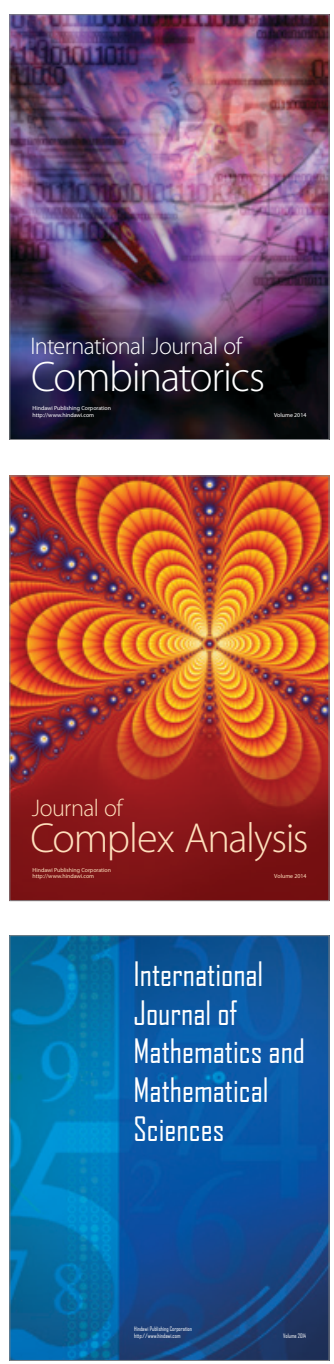
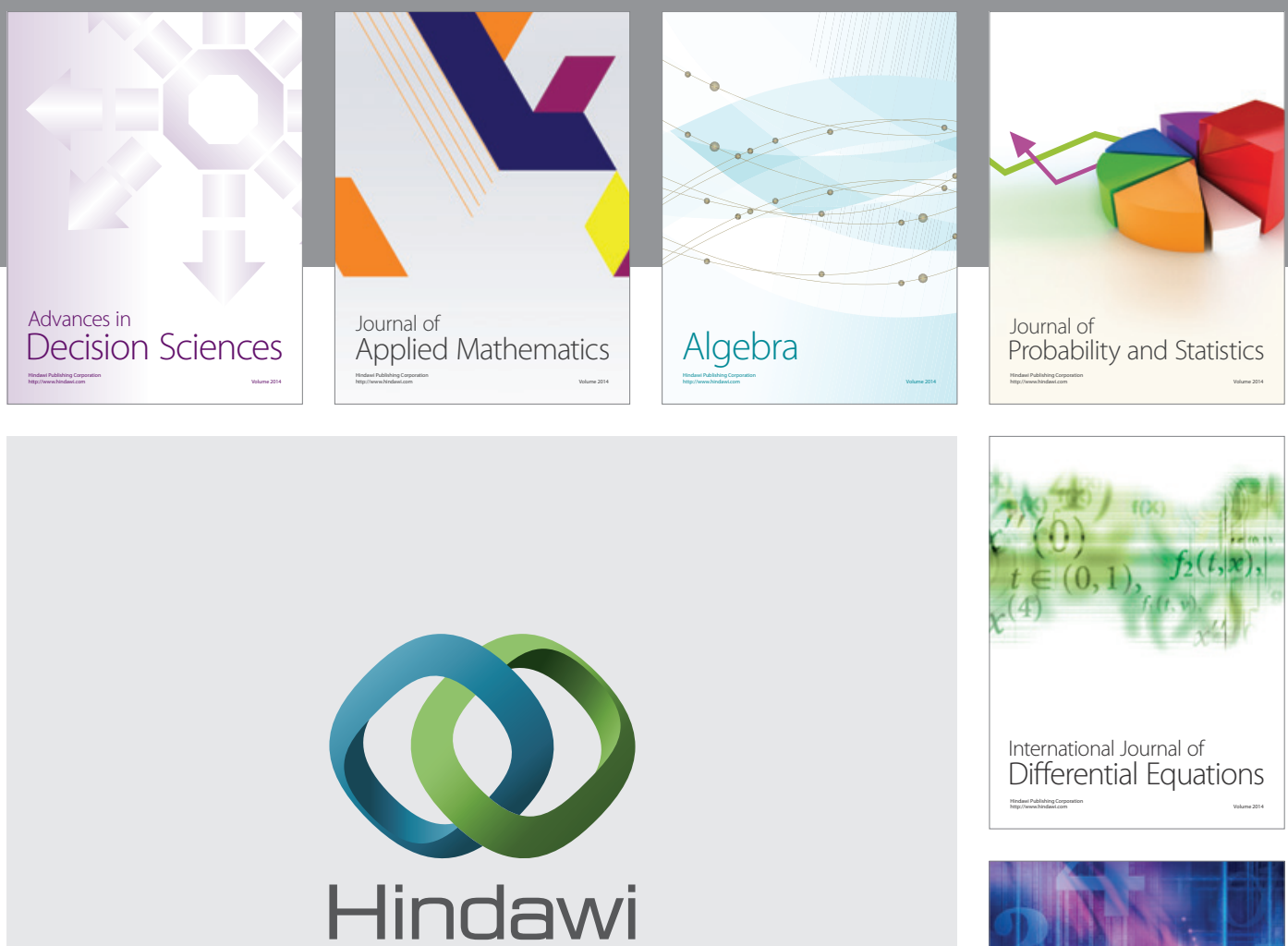

Submit your manuscripts at http://www.hindawi.com
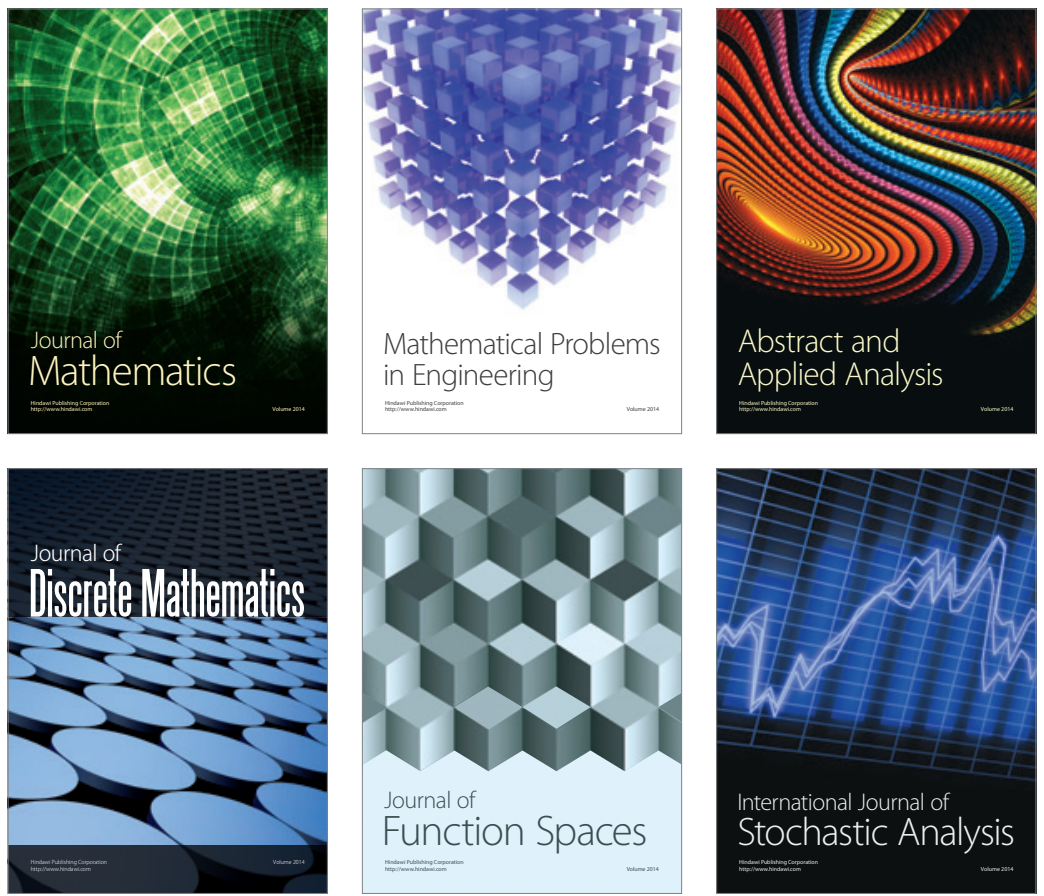

Journal of

Function Spaces

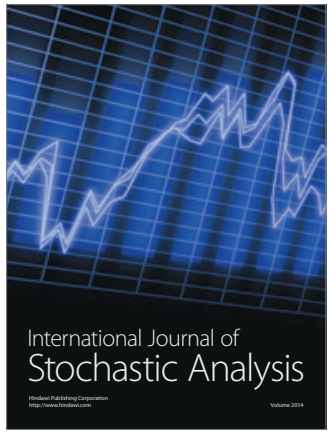

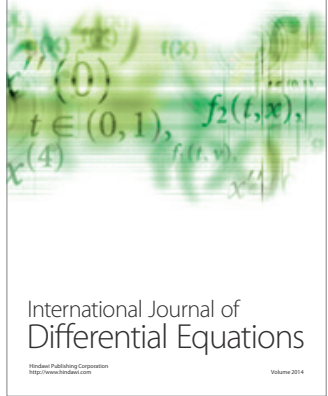
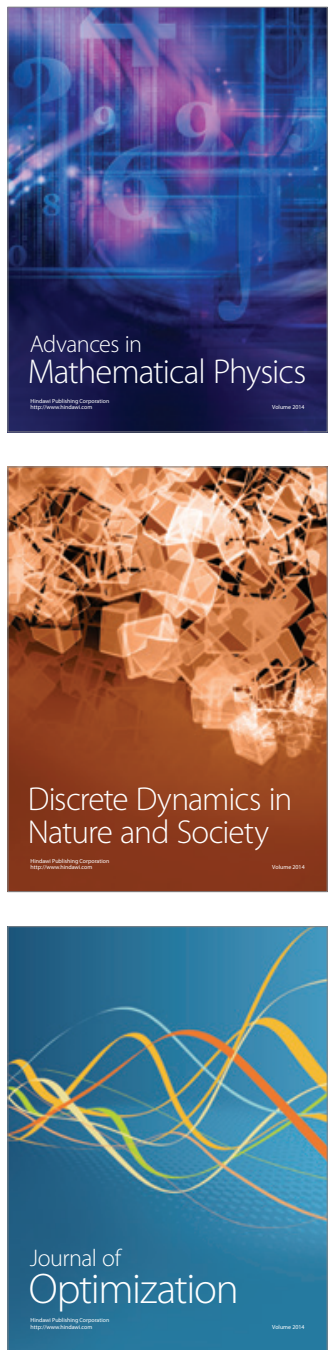